\title{
FRONT PROPAGATION IN NONLINEAR PARABOLIC EQUATIONS
}

\author{
EDUARD FEIREISL, DANIELLE HILHORST, HANA PETZELTOVÁ, AND PETER TAKÁČ
}

\begin{abstract}
We study existence and stability of travelling waves for nonlinear convection diffusion equations in the 1-D Euclidean space. The diffusion coefficient depends on the gradient in analogy with the $p$-Laplacian and may be degenerate. Unconditional stability is established with respect to initial data perturbations in $L^{1}(\mathbb{R})$.
\end{abstract}

Running head: Poincaré inequality and P.-S. condition

Keywords: Poincaré inequality, Palais-Smale condition, $p$-Laplacian, energy functional

2000 Mathematics Subject Classification: Primary 35J20, 35B45; Secondary 35P30, 46E35

E.F. acknowledges support from the Czech Science Foundation (GAČR) under the project P201-13-00522S in the framework of RVO: 67985840.

P.T. was partially supported by the grant TA 213/15-1 from the German Research Foundation (D.F.G.) of Germany. 


\section{INTRODUCTION}

The purpose of this article is to investigate the propagation of very simple travelling waves in a reaction-diffusion model. The model is the favorite Fisher-KPP equation (or Fisher-Kolmogorov equation) derived by R. A. FisheR [14] in 1937 and first mathematically analyzed by A. Kolmogorov, I. Petrovski, and N. Piscounov [18] in the same year. However, these original works $([14,18])$ consider solely linear diffusion and (sufficiently) smooth reaction. In our present work, we allow for both, a nonlinear diffusion operator and a nonsmooth reaction function. More precisely, we study the interaction between the (nonlinear) diffusion and the (nonsmooth) reaction; in paticular, their influence on the formation and the shape of a travelling wave connecting two stable (spatially constant) steady states.

We consider the following nonlinear evolutionary problem for an unknown function $u=u(x, t)$,

$$
\partial_{t} u=\operatorname{div}\left(\partial \Phi\left(\nabla_{x} u\right)\right)+f(u), \quad x \in \mathbb{R}^{N}, t>0,
$$

supplemented by the initial condition

$$
u(\cdot, 0)=u_{0} \quad \text { in } \mathbb{R}^{N}
$$

Here, $\Phi: \mathbb{R}^{N} \rightarrow \mathbb{R}, f: \mathbb{R} \rightarrow \mathbb{R}$, and $u_{0}: \mathbb{R}^{N} \rightarrow \mathbb{R}$ are given data as specified below. Roughly speaking, we assume that $\Phi$ is a continuously differentiable, convex functional on $\mathbb{R}^{N}$ with the Fréchet derivative $\partial \Phi: \mathbb{R}^{N} \rightarrow \mathbb{R}^{N}$, such that $\Phi$ is also radially symmetric of class $C^{2}\left(\mathbb{R}^{N} \backslash\{\mathbf{0}\}\right)$, its Hessian matrix $\partial^{2} \Phi(Z) \in \mathbb{R}^{N \times N}$ is positively definite at every point $Z \in \mathbb{R}^{N} \backslash\{\mathbf{0}\}$, and

$$
\left|\partial^{2} \Phi(Z)\right| \cdot|Z| \rightarrow 0 \quad \text { as }|Z| \rightarrow 0
$$

The nonlinear reaction function $f: \mathbb{R} \rightarrow \mathbb{R}$ is of the $\boldsymbol{K P} \boldsymbol{P}$-type (Kolmogorov-PetROVSKI-PISCOUNOV [18]); specifically,

$$
f \in C(\mathbb{R}), f(-1)=f(\mu)=f(1)=0, f<0 \text { in }(-1, \mu), f>0 \text { in }(\mu, 1) .
$$

Moreover, we assume that its integral

$$
F(r) \stackrel{\text { def }}{=} \int_{-1}^{r} f(s) \mathrm{d} s, \quad-1 \leq r \leq 1
$$

satisfies

$$
F(1)-F(r)=\int_{r}^{1} f(s) \mathrm{d} s>0 \quad \text { whenever }-1<r<1 .
$$

Taking the initial data $u_{0}: \mathbb{R}^{N} \rightarrow \mathbb{R}$ valued in the interval $[-1,1]$ between the extremal zeros $(=\mp 1)$ of $f$, i.e., $-1 \leq u_{0} \leq 1$, we are interested in the long-time behavior of solutions to problem (1.1), (1.2); in particular, in propagation of fronts separating the areas where $u$ approaches the limit values \pm 1 , respectively. 
A currently standard approach consists of introducing the (hyperbolic) change of variables $t \approx \frac{t}{\varepsilon}$ and $x \approx \frac{x}{\varepsilon}$ which leads us to the scaled problem

$$
\partial_{t} u_{\varepsilon}=\operatorname{div}\left(\partial \Phi\left(\varepsilon \nabla_{x} u_{\varepsilon}\right)\right)+\frac{1}{\varepsilon} f\left(u_{\varepsilon}\right), \quad x \in \mathbb{R}^{N}, t>0,
$$

for the unknown function $u_{\varepsilon}(x, t) \stackrel{\text { def }}{=} u\left(\frac{x}{\varepsilon}, \frac{t}{\varepsilon}\right)$, supplemented by the initial data

$$
u_{\varepsilon}(\cdot, 0)=u_{\varepsilon, 0} \text { in } \mathbb{R}^{N},
$$

see the survey by P. E. Souganidis [22]. A prototype example of (1.5) is the equation involving the $p$-Laplace operator $(p>1)$,

$$
\partial_{t} u_{\varepsilon}=\varepsilon^{p-1} \operatorname{div}\left(\left|\nabla_{x} u_{\varepsilon}\right|^{p-2} \nabla_{x} u_{\varepsilon}\right)+\frac{1}{\varepsilon} f\left(u_{\varepsilon}\right), \quad x \in \mathbb{R}^{N}, t>0 .
$$

Our aim is to examine the behavior of solutions $u_{\varepsilon}$ of problem (1.5), (1.6) as $\varepsilon \rightarrow 0+$. In particular, we extend the results of ZHAO and YI [24] for problem (1.7) with $p>2$ (the "degenerate case" of slow diffusion) to the "singular case" $1<p<2$ of fast diffusion.

1.1. Travelling waves. The asymptotic behavior of solutions $u_{\varepsilon}$ to problem (1.7) in the singular limit $\varepsilon \rightarrow 0+$ is well-understood in the nondegenerate case (slow diffusion case) $p=2(p>2)$ and also for the porous media type elliptic operator $\Delta u^{m}, m>1$, see Aronson and Weinberger [1], Chen [6], Feireisl [12], Fife and McLeod [13], and ZHAO and YI [24].

To begin, we decompose $\mathbb{R}^{N}$ into the closures of the following two regions:

$$
G_{-} \stackrel{\text { def }}{=}\left\{x \in \mathbb{R}^{N}: \text { there exists a neighborhood } U(x) \subset \mathbb{R}^{N} \text { of } x\right. \text { such that }
$$

$$
\left.\limsup _{\varepsilon \rightarrow 0+}\left(\sup _{y \in U(x)} u_{\varepsilon, 0}(y)\right)<\mu\right\}
$$

and

$$
\begin{array}{r}
G_{+} \stackrel{\text { def }}{=}\left\{x \in \mathbb{R}^{N}: \text { there exists a neighborhood } U(x) \subset \mathbb{R}^{N} \text { of } x\right. \text { such that } \\
\left.\qquad \limsup _{\varepsilon \rightarrow 0+}\left(\inf _{y \in U(x)} u_{\varepsilon, 0}(y)\right)>\mu\right\},
\end{array}
$$

where we assume that

$$
\bar{G}_{-} \cup \bar{G}_{+}=\mathbb{R}^{N}, \quad \text { and set } \quad \Gamma=\bar{G}_{-} \cap \bar{G}_{+} .
$$

Similarly to Barles, Bronsard, and Souganidis [3], we expect that (1.11) $u_{\varepsilon} \rightarrow-1$ uniformly in compact subsets of $\left\{(x, t) \in \mathbb{R}^{\mathbb{N}} \times \mathbb{R}_{+}: \operatorname{dist}[x, \Gamma]>c t\right\}$, $u_{\varepsilon} \rightarrow 1$ uniformly in compact subsets of $\left\{(x, t) \in \mathbb{R}^{\mathbb{N}} \times \mathbb{R}_{+}: \operatorname{dist}[x, \Gamma]<c t\right\}$, where $\mathbb{R}_{+} \stackrel{\text { def }}{=}[0, \infty)$ and "dist" stands for the signed distance,

$$
\operatorname{dist}[x, \Gamma]=\left\{\begin{aligned}
\inf \{|x-y|: y \in \Gamma\} & \text { for } x \in G_{-}, \\
-\inf \{|x-y|: y \in \Gamma\} & \text { for } x \in G_{+},
\end{aligned}\right.
$$


where $c$ is the front speed that can be determined as the speed of propagation of the traveling waves for the associated 1D problem.

Setting $u(x, t)=q(x-c t)$ in eq. (1.1) we have $\partial_{t} u=-c q_{x}$ and, thus, we look for solutions of the 1D problem

$$
\left\{\begin{array}{l}
\mathrm{d}_{x}\left[\partial \Phi\left(q_{x}\right)\right]+c q_{x}+f(q)=0 \quad \text { for } x \in \mathbb{R}, \\
q_{x} \leq 0 \text { in } \mathbb{R} \text { and } \quad \lim _{x \rightarrow-\infty} q(x)=1, \quad \lim _{x \rightarrow \infty} q(x)=-1,
\end{array}\right.
$$

normalized by the condition

$$
q(0)=\mu \text {. }
$$

As usual, we abbreviate the derivative $q_{x} \equiv \mathrm{d}_{x} q \equiv q^{\prime}$. We show that the front speed $c$ as well as the solution $q$ of (1.14), (1.15) are unique, and the asymptotic behavior of solutions to (1.5) is uniquely determined by $(1.11)$, (1.12). Since, by (1.14), we have

$$
\int_{-\infty}^{\infty}\left|q_{x}\right| \mathrm{d} x=\int_{-\infty}^{\infty}\left(-q_{x}\right) \mathrm{d} x=-\lim _{x \rightarrow \infty} q(x)+\lim _{x \rightarrow-\infty} q(x)=2
$$

and the limits $\lim _{x \rightarrow \pm \infty} \tilde{\Phi}\left(q_{x}\right)$ exist in $\mathbb{R}$ for

$$
\tilde{\Phi}(z) \stackrel{\text { def }}{=} \int_{0}^{z} \partial^{2} \Phi(s) s \mathrm{~d} s, \quad z \in \mathbb{R},
$$

where $\partial^{2} \Phi(s)>0$ for $s \in \mathbb{R} \backslash\{0\}$, so do the limits $\lim _{x \rightarrow \pm \infty} q_{x}$. Consequently, owing to $q_{x} \in L^{1}(\mathbb{R})$, we have also

$$
q_{x}(x) \rightarrow 0 \quad \text { as } x \rightarrow \pm \infty .
$$

Finally, multiplying eq. (1.14) by $q_{x}$ we get

$$
\mathrm{d}_{x}\left[\tilde{\Phi}\left(q_{x}\right)\right]+c\left|q_{x}\right|^{2}+\mathrm{d}_{x} F(q)=0 \quad \text { for } x \in \mathbb{R},
$$

and integrating from $-\infty$ to $+\infty$ we arrive at

$$
c \int_{-\infty}^{\infty}\left|q_{x}\right|^{2} \mathrm{~d} x=\int_{-1}^{1} f(q) \mathrm{d} q=F(1)-F(-1)=F(1) .
$$

Note that condition (1.4), i.e., $F(r)<F(1)$ for every $r \in(-1,1)$, forces $F(1) \geq$ $F(-1)=0$. In order to exclude the stationary solution $u(x, t)=q(x)$ with $c=0$, we will assume $F(1)>0$, in addition to (1.4), i.e.,

$$
F(1)=\int_{-1}^{1} f(s) \mathrm{d} s>0 .
$$

The paper is organized as follows. In the next section (Section 2) we recall some known results concerning solvability of problem (1.1), (1.2) and state our main result in Theorem 2.6. Section 3 is dedicated to the analysis of the travelling wave problem (1.14), (1.15). In particular, we show that problem (1.14), (1.15) admits a unique wave speed $c$ and a unique solution $q$ for a fairly general class of nonlinearities $\partial \Phi$ and $f$; see Proposition 3.1. This means that no solution to this problem exists for any other wave speed. Finally, the convergence claimed in (1.11), (1.12) is established in Section 4. 


\section{Preliminaries, Weak solutions, main Result}

We shall say that a function $u: \mathbb{R}^{N} \times \mathbb{R}_{+} \rightarrow \mathbb{R}$ is a weak solution to problem (1.1), (1.2) in $\mathbb{R}^{N} \times \mathbb{R}_{+}$if it belongs to the class

$$
\begin{aligned}
& u \in L^{\infty}\left(\mathbb{R}^{N} \times(0, \infty)\right), \quad u \in C_{\text {weak }}\left([0, T] \rightarrow L_{\text {loc }}^{1}\left(\mathbb{R}^{N}\right)\right) \quad \text { for every } T>0, \\
& \text { and } \quad \nabla_{x} u, \partial \Phi\left(\nabla_{x} u\right) \in L^{\infty}\left(\mathbb{R}^{N} \times(0, T) \rightarrow \mathbb{R}^{N}\right),
\end{aligned}
$$

and satisfies the integral identity

$$
\begin{aligned}
& \int_{\mathbb{R}^{N}} u(x, T) \psi(x, T) \mathrm{d} x-\int_{\mathbb{R}^{N}} u_{0}(x) \psi(x, 0) \mathrm{d} x \\
& =\int_{0}^{T} \int_{\mathbb{R}^{N}}\left[u \partial_{t} \psi-\partial \Phi\left(\nabla_{x} u\right) \cdot \nabla_{x} \psi+f(u) \psi\right] \mathrm{d} x \mathrm{~d} t
\end{aligned}
$$

required to hold for every test function $\psi \in C_{\mathrm{c}}^{\infty}\left(\mathbb{R}^{N} \times \mathbb{R}_{+}\right)$and for every $T \geq 0$. As usual, $C_{\mathrm{c}}^{\infty}\left(\mathbb{R}^{N} \times \mathbb{R}_{+}\right)$denotes the space of all infinitely many times differentiable functions $\psi$ : $\mathbb{R}^{N} \times \mathbb{R}_{+} \rightarrow \mathbb{R}$ with compact support $\left(\subset \mathbb{R}^{N} \times \mathbb{R}_{+}\right)$. For a weak solution to be well-defined, the function $u$ must obey the integrability conditions indicated above.

Analogously, we may define the (weak) sub- and supersolutions by changing the equality sign "=" in eq. (2.1) to " $\geq$ " and " $\leq$ ", respectively, and taking there nonnegative test functions $\psi$ only.

In fact, with a help from the "regularity" Proposition 2.2 below, which guarantees the (local Hölder-) continuity of a weak solution $u$ in $\mathbb{R}^{N} \times(0, \infty)$, we will construct a viscosity solution to problem (1.1), (1.2) in $\mathbb{R}^{N} \times \mathbb{R}_{+}$.

\subsection{Existence of weak solutions, comparison principle, uniqueness.}

- We assume that the function $\Phi: \mathbb{R}^{N} \rightarrow \mathbb{R}$ satisfies the following hypotheses:

$$
\begin{aligned}
& \left\{\begin{array}{r}
\Phi: \mathbb{R}^{N} \rightarrow \mathbb{R} \text { is radially symmetric, i.e., } \Phi(Z) \equiv \varphi(|Z|) \text { for every } Z \in \mathbb{R}^{N}, \\
\Phi \in C^{1}\left(\mathbb{R}^{N}\right) \cap C^{2}\left(\mathbb{R}^{N} \backslash\{0\}\right), \quad \varphi \in C^{1}\left(\mathbb{R}_{+}\right) \cap C^{2}((0, \infty)),
\end{array}\right. \\
& \left\{\begin{array}{l}
\varphi(0)=0, \quad \mathrm{~d}_{z} \varphi(0)=0, \quad \text { together with } \\
\mathrm{d}_{z} \varphi(z)>0 \quad \text { and } \quad \Lambda_{1} \leq \frac{z \cdot \mathrm{d}_{z, z}^{2} \varphi(z)}{\mathrm{d}_{z} \varphi(z)} \leq \Lambda_{2} \quad \text { for all } z \in(0, \infty), \\
\text { where } \Lambda_{1}, \Lambda_{2}>0 \text { are some positive constants. }
\end{array}\right.
\end{aligned}
$$

Moreover, there exists a continuous "modulus of continuity" function $\omega \in C\left(\mathbb{R}_{+}\right)$with $\omega(0)=0$, such that

$$
\begin{gathered}
\left|\mathrm{d}_{z, z}^{2} \varphi(s)-\mathrm{d}_{z, z}^{2} \varphi(y)\right| \leq \omega\left(\frac{|s-y|}{y}\right) \cdot \mathrm{d}_{z, z}^{2} \varphi(y) \\
\text { for all } s, y \in(0, \infty) \text { satisfying }|s-y|<\frac{1}{2} y .
\end{gathered}
$$


- Besides the KPP condition (1.3), condition (1.4), and $F(1)>0$, we assume that

$$
f: \mathbb{R} \rightarrow \mathbb{R} \quad \text { is Lipschitz continuous. }
$$

Remark 2.1. Condition (2.3) implies that $\varphi: \mathbb{R}_{+} \rightarrow \mathbb{R}$ is strictly monotone increasing and strictly convex. Moreover, the Euclidean norm $|\cdot|: \mathbb{R}^{N} \rightarrow \mathbb{R}$ is strictly convex (even uniformly convex). It is now an easy exercise to verify that also the function $\Phi=\varphi \circ|\cdot|: \mathbb{R}^{N} \rightarrow \mathbb{R}, \Phi(Z) \equiv \varphi(|Z|)$ for $Z \in \mathbb{R}^{N}$, must be strictly convex.

Hypotheses (2.3), (2.4) were introduced by G. M. Lieberman [20, 21]. In particular, both are satisfied for a finite sum of $p$-Laplace operators with different exponents $p=p_{i} \in$ $(1, \infty)$. Roughly speaking, hypothesis $(2.3)$ guarantees a priori bounds on $\left\|\nabla_{x} u\right\|_{L_{\mathrm{loc}}^{\infty}}$ in terms of $\|u\|_{L^{\infty}}$ and, in combination with (2.4), also the Hölder continuity of $\nabla_{x} u$ for any bounded weak solution $u$ of (1.1), (1.2), see G. M. LiEBERMAn [20, 21]. The implications of (2.3), (2.4) on the structural properties of $\varphi$ as well as other applications to the related elliptic problems are discussed by Breit, Stroffolini, and Verde [4].

2.1.1. A priori bounds. We quote the following crucial result on interior regularity estimates for equation (1.1) from G. M. LiEBERMAN [20], [21]:

Proposition 2.2. Let $f$ and $\Phi$ satisfy hypotheses (1.3), (1.4), and (2.2)-(2.5). Assume that $u$ is a weak solution of eq. (1.1) in a bounded open space-time cylinder

$$
Q_{R, T}=\left\{(x, t) \in \mathbb{R}^{N} \times \mathbb{R}_{+}:|x|<R, 0<t<T\right\},
$$

for some $R, T \in(0, \infty)$, belonging to the class

$$
u \in L^{\infty}\left(Q_{R, T}\right), \quad \nabla_{x} u \in L^{\infty}\left(Q_{R, T} \rightarrow \mathbb{R}^{N}\right) .
$$

(i) Then both $u, \nabla_{x} u$ are $\alpha$-Hölder continuous in the set

$$
Q_{R, T, \delta}=\left\{(x, t) \in Q_{R, T}:|x|<R-\delta, t>\delta\right\} \quad \text { for any (sufficiently small) } \delta>0 \text {, }
$$

$$
\|u\|_{C^{\alpha}\left(Q_{R, T, \delta}\right)}+\left\|\nabla_{x} u\right\|_{C^{\alpha}\left(Q_{R, T, \delta}\right)} \leq M
$$

where $\alpha$ and $M$ depend solely on the set of parameters

$$
\left[R, T, \delta, \Lambda_{1}, \Lambda_{2}, \partial_{z} \varphi(1), \omega,\|u\|_{L^{\infty}\left(Q_{R, T}\right)},\|f(u)\|_{L^{\infty}\left(Q_{R, T}\right)}\right] .
$$

(ii) If, in addition,

$$
u(\cdot, 0)=u_{0} \in C^{1+\beta}\left(\left\{x \in \mathbb{R}^{N}:|x| \leq R\right\}\right)
$$

then (2.6) holds also for $\delta=0$ in Part (i), i.e., in $Q_{R, T}$, with $\alpha$ and $M$ depending also on $\beta$ and $\left\|u_{0}\right\|_{C^{1+\beta}}$.

Proposition 2.2 yields an important corollary: 
Corollary 2.3. Let $f$ and $\Phi$ satisfy hypotheses (1.3), (1.4), and (2.2)-(2.5). Let $u$ be a weak solution to problem (1.1), (1.2) in the set $\mathbb{R}^{N} \times(0, \infty)$ belonging to the class

$$
u \in L^{\infty}\left(\mathbb{R}^{N} \times(0, \infty)\right), \quad-1 \leq u \leq 1, \quad \nabla_{x} u \in L_{\mathrm{loc}}^{\infty}\left(\mathbb{R}^{N} \times[0, \infty) \rightarrow \mathbb{R}^{N}\right),
$$

with the initial data

$$
\left\{\begin{array}{l}
u_{0} \in B U C\left(\mathbb{R}^{N}\right), \nabla_{x} u_{0} \in B U C\left(\mathbb{R}^{N} \rightarrow \mathbb{R}^{N}\right), \quad-1 \leq u_{0}(x) \leq 1 \text { for } x \in \mathbb{R}^{N} \\
\left|\nabla_{x} u_{0}(x)-\nabla_{x} u_{0}(y)\right| \leq L|x-y|^{\beta} \text { for all } x, y \in \mathbb{R}^{N} \text { and certain } \beta \in(0,1]
\end{array}\right.
$$

Then there is a constant $M \in \mathbb{R}_{+}$, depending solely on the quantities

$$
\Lambda_{1}, \Lambda_{2}, \mathrm{~d}_{z} \varphi(1), \omega, L, \beta,\left\|\nabla_{x} u_{0}\right\|_{L^{\infty}\left(\mathbb{R}^{N}\right)},
$$

such that

$$
\left|\nabla_{x} u(t, x)\right| \leq M \quad \text { for all } x \in \mathbb{R}^{N} \text { and } t \in \mathbb{R}_{+} .
$$

2.1.2. Existence of solutions. With the a priori estimates stated in Proposition 2.2 at hand, proving existence of a weak solution to problem (1.1), (1.2) is standard, at least for smooth initial data. More precisely, fixing the initial data $u_{0}$ in the regularity class (2.7), we may proceed in several steps, as follows:

Step 1. Without any loss of generality, in (2.3) we may assume

$$
0<\Lambda_{1}<1<\Lambda_{2}
$$

We perform a quadratic (Laplacian-type) regularization of the function $\Phi$ near the origin in $\mathbb{R}^{N}$ by replacing $\Phi$ by another $C^{2}$-smooth, radially symmetric function $\Phi_{\alpha} \in C^{2}\left(\mathbb{R}^{N}\right)$ $(\alpha>0)$, where

$$
\begin{aligned}
& \Phi_{\alpha}(Z) \equiv \varphi_{\alpha}(|Z|) \text { for every } Z \in \mathbb{R}^{N}, \quad \varphi_{\alpha} \in C^{2}\left(\mathbb{R}_{+}\right), \\
& \left\{\begin{array}{l}
\varphi_{\alpha}(0)=0, \quad \mathrm{~d}_{z} \varphi_{\alpha}(0)=0, \quad \text { together with } \\
\mathrm{d}_{z} \varphi_{\alpha}(z)>0 \quad \text { and } \quad \Lambda_{1} \leq \frac{z \cdot \mathrm{d}_{z, z}^{2} \varphi_{\alpha}(z)}{\mathrm{d}_{z} \varphi_{\alpha}(z)} \leq \Lambda_{2} \quad \text { for all } z>0,
\end{array}\right. \\
& \left\{\begin{array}{r}
\left|\mathrm{d}_{z, z}^{2} \varphi_{\alpha}(s)-\mathrm{d}_{z, z}^{2} \varphi_{\alpha}(y)\right| \leq \omega\left(\frac{|s-y|}{y}\right) \cdot \mathrm{d}_{z, z}^{2} \varphi_{\alpha}(y) \\
\text { for all } s, y \in(0, \infty) \text { satisfying }|s-y|<\frac{1}{2} y,
\end{array}\right. \\
& \frac{z \cdot \mathrm{d}_{z, z}^{2} \varphi_{\alpha}(z)}{\mathrm{d}_{z} \varphi_{\alpha}(z)}=1 \quad \text { for all } 0 \leq z \leq \frac{1}{\alpha}, \quad \text { and } \\
& \mathrm{d}_{z} \varphi_{\alpha} \rightarrow \mathrm{d}_{z} \varphi \quad \text { as } \alpha \searrow 0 \text {, uniformly on compact subsets of } \mathbb{R}_{+} \text {. }
\end{aligned}
$$

Here, the positive constants $0<\Lambda_{1}<1<\Lambda_{2}$ and the "modulus of continuity" function $\omega \in C\left(\mathbb{R}_{+}\right)$, with $\omega(0)=0$, are the same as in hypotheses (2.3) and (2.4); in particular, all of them are independent from $\alpha>0$.

We remark that hypothesis (2.12) is equivalent to

$$
\mathrm{d}_{z} \log \left(\frac{\mathrm{d}_{z} \varphi_{\alpha}(z)}{z}\right)=0 \quad \text { for all } 0 \leq z \leq \frac{1}{\alpha}
$$


which forces $\varphi_{\alpha}(z)=$ const $_{\alpha} \cdot z^{2}$ for all $0 \leq z \leq \frac{1}{\alpha}$, with a positive constant. Thus, $\varphi_{\alpha}$ is a qudratic regularization of the function $\varphi: \mathbb{R}_{+} \rightarrow \mathbb{R}$ near zero. This kind of regularization is typical in a construction of a viscosity solution to a quasilinear parabolic problem.

Step 2. Thanks to eq. (2.12), the resulting problem (1.1), (1.2) with $\Phi$ replaced by $\Phi_{\alpha}$ (defined in Step 1) is uniformly parabolic; thus, by virtue of the standard theory for parabolic equations from O. A. Ladyzhenskaya, V. A. Solonnikov, and N. N. URAL'TSEVA [19], it admits a unique (classical) solution $u_{\alpha}$ for any fixed $\alpha>0$.

Step 3. Since $f$ vanishes at \pm 1 , the constant functions $u(x, t) \equiv \pm 1$ are (classical) solutions of eq. (1.1). Thanks to $-1 \leq u_{\alpha}(\cdot, 0)=u_{0} \leq 1$ in the initial condition (1.2), we may apply the classical version of the (parabolic weak) comparison principle to deduce that also

$$
-1 \leq u_{\alpha}(t, x) \leq 1 \quad \text { for all } x \in \mathbb{R}^{N}, t \geq 0
$$

Step 4. The family of classical solutions $\left\{u_{\alpha}\right\}_{\alpha>0}$ satisfies the hypotheses of Proposition 2.2 and Corollary 2.3, with the parameters independent from $\alpha$. Consequently, it is easy to pass to the limit for $\alpha \searrow 0$, at least for a suitable subsequence, to deduce the following existence result:

Proposition 2.4. Let the functions $f$ and $\Phi$ satisfy hypotheses (1.3), (1.4), and (2.2)(2.5). Then for any initial data $u_{0}$ satisfying (2.7), the Cauchy problem (1.1), (1.2) admits a weak solution $u$ in the class

$$
\begin{aligned}
& -1 \leq u(t, x) \leq 1, \quad\left|\nabla_{x} u(t, x)\right| \leq M \text { for all } x \in \mathbb{R}^{N}, t \geq 0, \\
& u, \nabla_{x} u \text { belong to } C^{\kappa}(\mathcal{K}), \kappa \in(0,1), \text { for any compact set } \mathcal{K} \subset \mathbb{R}^{N} \times[0, \infty) .
\end{aligned}
$$

2.1.3. Admissible weak solutions. The construction procedure carried over in the preceding paragraph inspires the following definition.

Definition 2.5. We say that $u$ is an admissible weak solution to problem (1.1), (1.2), if there exists a sequence of regularized functions $\varphi_{\alpha_{n}}(n=1,2,3, \ldots)$ enjoying properties (2.10)-(2.13) and a sequence of initial data $u_{\alpha_{n}, 0}$ belonging to the regularity class (2.7), such that

$$
\begin{aligned}
& u_{\alpha_{n}, 0} \rightarrow u_{0} \quad \text { uniformly on compact sets in } \mathbb{R}^{N} \text {, as } n \rightarrow \infty ; \\
& u_{\alpha_{n}} \rightarrow u \quad \text { uniformly on compact sets in } \mathbb{R}^{N} \times[0, \infty),
\end{aligned}
$$

where $\alpha_{n} \searrow 0$ as $n \nearrow \infty$, and each $u_{\alpha_{n}}$ is the classical solution of problem (1.1), (1.2) corresponding to $\Phi=\Phi_{\alpha_{n}}$ and $u_{0}=u_{\alpha, 0}$.

It can be shown that the admissible weak solutions coincide with the standard viscosity solutions introduced for continuous initial data by CRANDALL, ISHII, and Lions [7] as soon as $\varphi \in C^{2}[0, \infty)$. As such, they satisfy the parabolic weak comparison principle and, consequently, are uniquely determined by the initial data; see Y. GiGA et al. [15]. 
Unfortunately, the singular case $\mathrm{d}_{z, z}^{2} \varphi \rightarrow \infty$ for $z \searrow 0$, that includes the $p$-Laplace operator with $1<p<2$, does not fit into the framework of [15], so that the mere definition of the concept of viscosity solution requires some nontrivial modifications, see Judtinen, Lindqvist, and Manfredi [17]. Although the results and techniques used by DiBenedetto and Herrero $[8,9]$ provide the uniqueness of weak solutions for $p$ Laplace-like operators with $1<p<2$ and $p \geq 2$, respectively, a general uniqueness theorem that would cover all cases allowed by hypotheses (2.3), (2.4) does not seem to be easily available in the existing literature. Note, however, that a well-posedness theory can be established by the method of monotone operators (see, e.g., V. BARBU [2] or H. BRÉzis [5]) as soon as the initial data approach one of the zeros of the function $f$ as $|x| \rightarrow \infty$.

2.2. Main result. We will show in Section 3 that the traveling wave problem (1.14), (1.15) admits a unique solution pair $[q, c]$. Accordingly, our main result may be stated as follows:

Theorem 2.6. Let the functions $f$ and $\Phi$ satisfy hypotheses (1.3), (1.4), $F(1)>0$, and (2.2)-(2.5). Assume that $\left\{u_{\varepsilon}\right\}_{\varepsilon>0}$ is a family of admissible weak solutions of the Cauchy problem (1.5), (1.6), with the initial data $u_{\varepsilon}(\cdot, 0)=u_{\varepsilon, 0}: \mathbb{R}^{N} \rightarrow \mathbb{R}$ satisfying

$$
\begin{array}{r}
-1 \leq u_{\varepsilon, 0}(x) \leq 1 \quad \text { and } \quad\left|\nabla_{x} u_{\varepsilon, 0}(x)\right| \leq \operatorname{const}_{\varepsilon} \quad \text { for all } x \in \mathbb{R}^{N}, \quad \text { and } \\
\left|\nabla_{x} u_{\varepsilon, 0}(x)-\nabla_{x} u_{\varepsilon, 0}(y)\right| \leq \operatorname{const}_{\varepsilon} \cdot|x-y|^{\beta} \quad \text { for all } x, y \in \mathbb{R}^{N},
\end{array}
$$

where $\beta \in(0,1)$ is a constant independent from $\varepsilon>0$. Let $G_{-}$and $G_{+}$be the sets introduced in eqs. (1.8) and (1.9), respectively, such that eq. (1.10) holds, i.e., $\bar{G}_{-} \cup \bar{G}_{+}=$ $\mathbb{R}^{N}$. Denote $\Gamma=\bar{G}_{-} \cap \bar{G}_{+}$.

Then, as $\varepsilon \searrow 0$, we have

$$
\begin{gathered}
u_{\varepsilon} \rightarrow-1 \quad \text { uniformly in compact subsets of }\{(x, t): \operatorname{dist}[x, \Gamma]>c t\}, \\
u_{\varepsilon} \rightarrow 1 \quad \text { uniformly in compact subsets of }\{(x, t): \operatorname{dist}[x, \Gamma]<c t\},
\end{gathered}
$$

with the front speed $c$ being uniquely determined by eqs. (1.14), (1.15), c > 0, where dist stands for the signed distance introduced in (1.13).

The remaining part of the paper is devoted to the proof of Theorem 2.6.

\section{TRAVELling WAVES}

In this section we establish the following result on the existence and uniqueness of travelling waves in problem (1.14), (1.15).

Proposition 3.1. If the functions $f$ and $\Phi$ satisfy hypotheses (1.3), (1.4), and (2.2)(2.5), then problem (1.14), (1.15) admits a unique solution $[q, c]$. This solution satisfies 
also

$$
\begin{aligned}
& q_{x} \equiv \mathrm{d}_{x} q \in L^{1}(\mathbb{R}) \cap L^{\infty}(\mathbb{R}), \\
& q_{x}(x)<0 \text { for every } x \in \mathbb{R} \text { such that }-1<q(x)<1, \quad \text { and } \\
& c=\left(\int_{-\infty}^{\infty}\left|q_{x}\right|^{2} \mathrm{~d} x\right)^{-1} \int_{-1}^{1} f(s) \mathrm{d} s \geq 0 .
\end{aligned}
$$

In particular, we have $c>0$ if and only if $F(1)=\int_{-1}^{1} f(u) \mathrm{d} u>0$.

We will see that the proof of this proposition follows directly from a combination of Lemma 3.9 (existence) and Proposition 3.8 (uniqueness) established below. The continuous dependence of the travelling wave $q$ and the speed $c$ upon the given data, combined with a standard compactness argument (Arzelà-Ascoli's theorem), enables us to apply a continuity (convergence) result from PH. HARTMAN's monograph [16, Theorem 2.1, p. 94] to establish an approximation and continuity result for problem (1.14), (1.15) stated in the next lemma.

To formulate this result, let us consider the following family of analogous problems parametrized by $\alpha \in(0,1)$, for an unknown pair $\left[q_{\alpha}, c_{\alpha}\right]$ (cf. eqs. (2.9)-(2.13)):

$$
\left\{\begin{array}{l}
\mathrm{d}_{x}\left[\partial \Phi_{\alpha}\left(\mathrm{d}_{x} q_{\alpha}\right)\right]+c_{\alpha} \cdot \mathrm{d}_{x} q_{\alpha}+f_{\alpha}\left(q_{\alpha}\right)=0 \quad \text { for } x \in \mathbb{R}, \\
\mathrm{d}_{x} q_{\alpha} \leq 0 \text { in } \mathbb{R} \text { and } \lim _{x \rightarrow-\infty} q_{\alpha}(x)=b_{\alpha}, \quad \lim _{x \rightarrow \infty} q_{\alpha}(x)=a_{\alpha},
\end{array}\right.
$$

where $-\infty<a_{\alpha}<b_{\alpha}<\infty$, with $q_{\alpha}$ normalized by the condition

$$
q_{\alpha}(0)=\mu_{\alpha}, \quad a_{\alpha}<\mu_{\alpha}<b_{\alpha} .
$$

The existence and uniqueness of the pair $\left[q_{\alpha}, c_{\alpha}\right]$ follow in the same way as those of $[q, c]$, cf. Proposition 3.1 above.

Lemma 3.2. Let $\left\{f_{\alpha}\right\}_{\alpha>0}$ be a family of uniformly Lipschitz-continuous functions $f_{\alpha}$ : $\mathbb{R} \rightarrow \mathbb{R}$, such that

$$
\begin{aligned}
& L \stackrel{\text { def }}{=} \sup _{\alpha>0}\left\|f_{\alpha}^{\prime}\right\|_{L^{\infty}(\mathbb{R})}<\infty ; \\
& \left\{\begin{array}{r}
-\infty<a_{\alpha}<\mu_{\alpha}<b_{\alpha}<\infty, \quad f_{\alpha}\left(a_{\alpha}\right)=f\left(\mu_{\alpha}\right)=f\left(b_{\alpha}\right)=0 \\
\text { with } \quad f_{\alpha}<0 \text { in }\left(a_{\alpha}, \mu_{\alpha}\right), \quad f_{\alpha}>0 \text { in }\left(\mu_{\alpha}, b_{\alpha}\right) ;
\end{array}\right. \\
& \left\{\begin{array}{l}
F_{\alpha}(r) \stackrel{\text { def }}{=} \int_{a_{\alpha}}^{r} f_{\alpha}(s) \mathrm{d} s \quad \text { satisfies } \\
F_{\alpha}\left(b_{\alpha}\right)-F_{\alpha}(r)=\int_{r}^{b_{\alpha}} f_{\alpha}(s) \mathrm{d} s>0 \quad \text { whenever } a_{\alpha}<r<b_{\alpha} ;
\end{array}\right. \\
& \left\{\begin{array}{l}
a_{\alpha} \rightarrow-1, \quad \mu_{\alpha} \rightarrow \mu, \quad b_{\alpha} \rightarrow 1, \quad \text { and } \\
f_{\alpha} \rightarrow f \text { locally uniformly in } C(\mathbb{R}) \quad \text { as } \alpha \searrow 0,
\end{array}\right.
\end{aligned}
$$

and the limit function $f$ satisfies all conditions in (1.3), $-1<\mu<1$, together with condition (1.4). Similarly, we assume that the family $\left\{\Phi_{\alpha}\right\}_{\alpha>0}$ satisfies all hypotheses in 
(2.9)-(2.13). Finally, let $\left[q_{\alpha}, c_{\alpha}\right](\alpha>0)$ be the uniquely determined family of solutions to problem (3.1), (3.2) satisfying

$$
\lim _{x \rightarrow-\infty} q_{\alpha}(x)=b_{\alpha}, \quad \lim _{x \rightarrow \infty} q_{\alpha}(x)=a_{\alpha}, \quad \text { and } \quad q_{\alpha}(0)=\mu_{\alpha},
$$

by Proposition 3.1. Then we have

$$
\begin{aligned}
& \left\|\mathrm{d}_{x} q_{\alpha}\right\|_{L^{1}(\mathbb{R})}+\left\|\mathrm{d}_{x} q_{\alpha}\right\|_{L^{\infty}(\mathbb{R})} \leq \text { const } \quad \text { together with } \\
& q_{\alpha} \rightarrow q \text { uniformly in } C(\mathbb{R}) \text { and } c_{\alpha} \rightarrow c \text { as } \alpha \searrow 0,
\end{aligned}
$$

where $[q, c]$ is the unique solution of (1.14), (1.15) corresponding to the limit functions $f$ and $\Phi$, by Proposition 3.1.

We begin with the proof of Proposition 3.1. Let us recall that we investigate monotone (decreasing) travelling waves in the degenerate second-order parabolic problem (1.1) of a "generalized" Fisher-KPP type. This task reduces to finding travelling waves in the following degenerate second-order parabolic problem reduced to one space dimension:

$$
\left\{\begin{aligned}
\partial_{t} u & =\partial_{x}\left(\partial \Phi\left(\partial_{x} u\right)\right)+f(u), \quad(x, t) \in \mathbb{R}^{1} \times \mathbb{R}_{+}, \\
u(x, t) & =q(x-c t) \quad \text { for some constant } c \in \mathbb{R}
\end{aligned}\right.
$$

Here, $\Phi: \mathbb{R}^{1} \rightarrow \mathbb{R}$ is an continuously differentiable, even convex function satisfying all hypotheses in (2.2) and (2.3), with the derivative $\partial \Phi \equiv \Phi^{\prime}: \mathbb{R}^{1} \rightarrow \mathbb{R}$.

For example, we may take $\Phi(z)=\frac{1}{p}|z|^{p}$ for $z \in \mathbb{R}$, where $1<p<\infty$ is a fixed number, so that $\partial \Phi(z) \equiv \Phi^{\prime}(z)=|z|^{p-2} z$ and $\partial^{2} \Phi(z) \equiv \Phi^{\prime \prime}(z)=(p-1)|z|^{p-2}$ for $z \in \mathbb{R}$.

Recall that $f: \mathbb{R} \rightarrow \mathbb{R}$ is assumed to be Lipschitz-continuous, by (2.5), and, most importantly, it satisfies the KPP condition (1.3), that is, $f( \pm 1)=f(\mu)=0$ for some $-1<\mu<1$, together with $f(s)<0$ for every $s \in(-1, \mu), f(s)>0$ for every $s \in(\mu, 1)$, and also condition (1.4), specifically

$$
F(1)-F(r)=\int_{r}^{1} f(s) \mathrm{d} s>0 \quad \text { whenever }-1<r<1 .
$$

Furthermore, by Proposition 3.1, we have $F(1)>0$ if and only if $c>0$.

Remark 3.3. An important special case of the reaction function $f$ is $f(s)=F^{\prime}(s)$ where $-F: \mathbb{R} \rightarrow \mathbb{R}$ is a "generalized" double-well potential ([3, eq. (0.2)]), such that

$$
\left\{\begin{aligned}
F^{\prime}(s) \equiv f(s) & =2(s-\mu)\left(1-s^{2}\right)=-2(s+1)(s-\mu)(s-1) \quad \text { for } s \in \mathbb{R} ; \\
F(-1) & =0,
\end{aligned}\right.
$$

whence

$$
\left\{\begin{array}{r}
-F(s)=\left(s^{2}-1\right)(s-\mu)^{2}-\frac{1}{2}(s-\mu)^{4}-\frac{2}{3} \mu(s-\mu)^{3}-F(\mu) \\
\text { for } s \in \mathbb{R}, \quad \text { where } \quad F(\mu)=-\frac{1}{2}(1+\mu)^{3}\left(1-\frac{\mu}{3}\right)<0 .
\end{array}\right.
$$


We have also $F(1)=-F(-\mu)+F(\mu)=-\frac{8}{3} \mu$ or

$$
\begin{aligned}
F(1) & =\int_{-1}^{1} f(s) \mathrm{d} s=2 \int_{0}^{1}[(s-\mu)+(-s-\mu)]\left(1-s^{2}\right) \mathrm{d} s \\
& =-4 \mu \int_{0}^{1}\left(1-s^{2}\right) \mathrm{d} s=-\frac{8}{3} \mu .
\end{aligned}
$$

In particular, condition (1.4) holds if and only if $\mu \leq 0$.

Assuming that the travelling wave takes the form $u(x, t)=q(x-c t),(x, t) \in \mathbb{R} \times \mathbb{R}_{+}$, with $q: \mathbb{R} \rightarrow \mathbb{R}$ being continuously differentiable and satisfying $q^{\prime}(x)<0$ at every point $x \in \mathbb{R}$ such that $-1<q(x)<1$, below, we are able to find a first integral for the second-order equation for $q$; cf. eq. (1.14):

$$
\mathrm{d}_{x}\left(\partial \Phi\left(\mathrm{d}_{x} q\right)\right)+c \cdot \mathrm{d}_{x} q+f(q)=0, \quad x \in \mathbb{R} .
$$

We will use the following (abuse of) notation exclusively throughout the remaining part of this section; it will not intervene with the notation for the function $\varphi=\varphi(|Z|)=$ $\Phi(Z)$ of $Z \in \mathbb{R}^{N}$ introduced in Section 2, eq. (2.2). This time, let us denote $\varphi=\partial \Phi \equiv \Phi^{\prime}$. By the properties (2.2) and (2.3) of $\Phi$ recalled above, $\varphi: \mathbb{R} \rightarrow \mathbb{R}$ is a continuous, strictly monotone increasing, odd function; hence, $\varphi(0)=0$. Moreover, we have $\varphi(s) \rightarrow \pm \infty$ as $s \rightarrow \pm \infty$, respectively. Consequently,

$$
\Phi(s) \stackrel{\text { def }}{=} \int_{0}^{s} \varphi(\xi) \mathrm{d} \xi, \quad \text { for } s \in \mathbb{R}
$$

is a continuously differentiable, strictly convex, even function, with $\Phi(0)=0$ and $\Phi(s) /|s|$ $\rightarrow+\infty$ for $s \rightarrow \pm \infty$. We denote by $\Psi: \mathbb{R} \rightarrow \mathbb{R}$ the convex conjugate function associated with $\Phi$, that is,

$$
\Psi(t) \stackrel{\text { def }}{=} \sup _{s \in \mathbb{R}}(s t-\Phi(s)) \quad \text { for } t \in \mathbb{R}
$$

Then, by the general theory for pairs of convex conjugate functions, also $\Psi$ is continuously differentiable, strictly convex, and even, with $\Psi(0)=0$ and $\Psi(t) /|t| \rightarrow+\infty$ for $t \rightarrow \pm \infty$. Its derivative $\psi \stackrel{\text { def }}{=} \Psi^{\prime}: \mathbb{R} \rightarrow \mathbb{R}$ is continuous, strictly monotone increasing, and odd. Moreover, the functions $\varphi, \psi: \mathbb{R} \rightarrow \mathbb{R}$ are each other's inverse, i.e., $\psi=\varphi_{-1}$ and $\varphi=\psi_{-1}$. We refer the reader to the monograph by I. EkEland and R. TEmam [10, Part 1] for details about convex conjugate functions.

Following the main ideas from R. EnguiçA, A. Gavioli, and L. Sanchez [11, Sect. 1], we make the substitution

$$
v \stackrel{\text { def }}{=}-\varphi\left(\mathrm{d}_{x} q\right)>0, \quad \text { where } \quad \mathrm{d}_{x} q \equiv q_{x} \equiv \frac{\mathrm{d} q}{\mathrm{~d} x}<0,
$$

whence

$$
\mathrm{d}_{x} q=-\varphi_{-1}(v)=-\psi(v)<0
$$


and consequently look for $v=v(q)$ as a function of $q \in(-1,1)$ that satisfies the following differential equation obtained from eq. (3.9):

$$
-\frac{\mathrm{d} v}{\mathrm{~d} q} \cdot \frac{\mathrm{d} q}{\mathrm{~d} x}+c \frac{\mathrm{d} q}{\mathrm{~d} x}+f(q)=0, \quad x \in \mathbb{R},
$$

that is, with a help from (3.12),

$$
\frac{\mathrm{d} v}{\mathrm{~d} q} \cdot \psi(v)-c \psi(v)+f(q)=0, \quad q \in(-1,1) .
$$

Finally, we make the substitution $y \stackrel{\text { def }}{=} \Psi(v)>0$, thus arriving at

$$
\frac{\mathrm{d} y}{\mathrm{~d} q}-c \psi\left(\Psi_{-1}(y)\right)+f(q)=0, \quad q \in(-1,1) .
$$

Here, $\Psi_{-1} \equiv\left(\left.\Psi\right|_{\mathbb{R}_{+}}\right)_{-1}: \mathbb{R}_{+} \rightarrow \mathbb{R}$ stands for the inverse function of $\Psi$ restricted to the domain $\mathbb{R}_{+} \stackrel{\text { def }}{=}[0, \infty)$ and, thus, denoted by $\left.\Psi\right|_{\mathbb{R}_{+}}$. In order to avoid possible confusion between the unknown function $q(x)$ of $x \in \mathbb{R}$ and the independent variable $q \in$ $(-1,1)$, we prefer to replace the latter by $r \in(-1,1)$. This means that the unknown function $y:(-1,1) \rightarrow(0, \infty)$ of $r$,

$$
y=\Psi(v)=\Psi\left(\varphi\left(\left|\mathrm{d}_{x} q\right|\right)\right)>0,
$$

must satisfy the following differential equation:

$$
\frac{\mathrm{d} y}{\mathrm{~d} r}-c \psi\left(\Psi_{-1}\left(y^{+}\right)\right)+f(r)=0, \quad r \in(-1,1)
$$

where $y^{+} \stackrel{\text { def }}{=} \max \{y, 0\}$ for $y \in \mathbb{R}$. Since we require that $q=q(x)$ be sufficiently smooth, at least continuously differentiable, with $q_{x}(x) \equiv q^{\prime}(x) \rightarrow 0$ as $x \rightarrow \pm \infty$, the function $y=y(r)$ must satisfy the boundary conditions

$$
y(-1)=y(1)=0 .
$$

Recalling the substitution $y=\Psi(v)>0$ for $v>0$, i.e., $v=\Psi_{-1}(y)$, from eq. (3.13) we deduce the following equivalent form of eq. (3.15) for the unknown function $v=v(r)$,

$$
\frac{\mathrm{d} v}{\mathrm{~d} r}-c+\frac{f(r)}{\psi(v)}=0, \quad r \in(-1,1)
$$

Furthermore, boundary conditions (3.16) for $y=y(r)$ become

$$
v(-1)=v(1)=0 \text {. }
$$

The following remark on the value of $F(1)(\geq 0)$ is in order. We recall that $f(r)$ satisfies the KPP condition (1.3), together with condition (1.4).

Remark 3.4. Since the integrand $f:(-1,1) \rightarrow \mathbb{R}$ in the function $F(r)$, used in (1.4) for $r \in(-1,1)$, is continuous and absolutely integrable over $(-1,1)$, we conclude that $F:[-1,1] \rightarrow \mathbb{R}$ is absolutely continuous. In particular, ineq. (1.4) forces $F(1) \geq 0$. We will see later that the case $F(1)=0$ guarantees the existence of a stationary solution to problem (3.7), i.e., $c=0$, whereas the case $F(1)>0$ renders a travelling wave, i.e., $c \neq 0$; more precisely, $c>0$, cf. Proposition 3.1 above. Indeed, both, the stationary solution (for 
$c=0$ ) and the travelling wave (for $c>0$ ) will be obtained from eq. (3.15) by means of the transformation defined by eqs. (3.12) and (3.14).

Recalling Remark 3.3, for the quartic double-well potential $-F$ given by eq. (3.8) we have

$$
c=\left(\int_{-\infty}^{\infty}\left|q_{x}\right|^{2} \mathrm{~d} x\right)^{-1} \int_{-1}^{1} f(s) \mathrm{d} s=-\frac{8}{3} \mu\left(\int_{-\infty}^{\infty}\left|q_{x}\right|^{2} \mathrm{~d} x\right)^{-1} .
$$

In order to investigate equation (3.15) (and (3.17), as well), we begin with the following more general differential equation than eq. (3.15), namely,

$$
\frac{\mathrm{d} y}{\mathrm{~d} r}-c H\left(y^{+}\right)=-f(r), \quad r \in(-1,1)
$$

where $H: \mathbb{R}_{+} \rightarrow \mathbb{R}$ is a continuous, strictly monotone increasing function with $H(0)=0$. In eq. (3.15), this means $H=\psi \circ \Psi_{-1}$ on $\mathbb{R}_{+}$.

The positivity condition (1.4) for $r \in(-1,1)$ starts from the terminal value of $r$ $(r=1)$. This would cause serious difficulties with notation and the uniqueness for a number of initial value problems that we are going to treat; namely, we would be forced to treat them as terminal value problems. Therefore, we make the substitution $z(r)=y(-r)$ for $-1 \leq r \leq 1$ (reflection about 0 ) for the unknown function $y$ and, consequently, look for a continuously differentiable solution $z:[-1,1] \rightarrow \mathbb{R}$ to the following Dirichlet boundary value problem equivalent to eq. (3.19),

$$
\frac{\mathrm{d} z}{\mathrm{~d} r}+c H\left(z^{+}\right)=f(-r), \quad r \in(-1,1) ; \quad z(-1)=z(1)=0,
$$

where $c \in \mathbb{R}$ is also an unknown constant to be determined. As usual, we apply the shooting method for solutions $z:[-1,1] \rightarrow \mathbb{R}$ using the initial condition $z(-1)=0$. We determine the constant $c \in \mathbb{R}$ such that also $z(1)=0$ hold.

The following comparison lemma is standard; cf. PH. Hartman [16, Corollary 4.2, p. 27].

Lemma 3.5. Let $c \in \mathbb{R}$ satisfy $c \geq 0$ and assume that $z_{1}, z_{2}:[a, b] \rightarrow \mathbb{R}$ are two absolutely continuous functions on some interval $[a, b] \subset \mathbb{R}$, such that $-1 \leq a<b \leq 1$, and the following inequality holds for almost every $r \in(a, b)$ :

$$
\frac{\mathrm{d} z_{1}}{\mathrm{~d} r}+c H\left(z_{1}^{+}\right) \leq \frac{\mathrm{d} z_{2}}{\mathrm{~d} r}+c H\left(z_{2}^{+}\right) .
$$

If $z_{1}(a) \leq z_{2}(a)$ then $z_{1}(r) \leq z_{2}(r)$ holds for all $r \in[a, b]$.

Proof. Rewriting ineq. (3.21) for the difference $z_{1}-z_{2}$, we have

$$
\frac{\mathrm{d}}{\mathrm{d} r}\left(z_{1}-z_{2}\right)+c\left(H\left(z_{1}^{+}\right)-H\left(z_{2}^{+}\right)\right) \leq 0 \quad \text { for a.e. } r \in(a, b) .
$$


Multiplying this difference by $\left(z_{1}-z_{2}\right)^{+}$, we arrive at

$$
\begin{array}{r}
\frac{1}{2} \cdot \frac{\mathrm{d}}{\mathrm{d} r}\left[\left(z_{1}-z_{2}\right)^{+}\right]^{2}+c\left(H\left(z_{1}^{+}\right)-H\left(z_{2}^{+}\right)\right)\left(z_{1}-z_{2}\right)^{+} \leq 0 \\
\text { for a.e. } r \in(a, b) .
\end{array}
$$

Taking into account that $c \geq 0$ and $s \mapsto H\left(s^{+}\right): \mathbb{R} \rightarrow \mathbb{R}$ is a monotone increasing function, with $H\left(s^{+}\right)=0$ for $s \leq 0$, we conclude that the second summand on the left-hand side of eq. (3.22) is nonnegative, which yields

$$
\frac{1}{2} \cdot \frac{\mathrm{d}}{\mathrm{d} r}\left[\left(z_{1}-z_{2}\right)^{+}\right]^{2} \leq 0 \quad \text { for a.e. } r \in(a, b) .
$$

Consequently, $r \mapsto\left[\left(z_{1}(r)-z_{2}(r)\right)^{+}\right]^{2}:[a, b] \rightarrow \mathbb{R}$ is a nonnegative, monotone decreasing function that vanishes at $r=a$; hence, it must vanish identically on the whole of $[a, b]$.

The lemma is proved.

Lemma 3.5 has the following easy, but very useful corollary.

Corollary 3.6. Let $c_{1}, c_{2} \in \mathbb{R}$ satisfy $c_{1} \geq c_{2}$ and $c_{1} \geq 0$. Assume that $z_{1}, z_{2}:[a, b] \rightarrow \mathbb{R}$ are two absolutely continuous functions on some interval $[a, b] \subset \mathbb{R}$, such that $-1 \leq a<$ $b \leq 1$, and the following inequalities hold for almost every $r \in(a, b)$ :

$$
\begin{aligned}
& \frac{\mathrm{d} z_{1}}{\mathrm{~d} r}+c_{1} H\left(z_{1}^{+}\right) \leq f(-r), \\
& \frac{\mathrm{d} z_{2}}{\mathrm{~d} r}+c_{2} H\left(z_{2}^{+}\right) \geq f(-r) .
\end{aligned}
$$

If $z_{1}(a) \leq z_{2}(a)$ then $z_{1}(r) \leq z_{2}(r)$ holds for all $r \in[a, b]$.

Proof. Inequalities (3.23) and (3.24) guarantee that (3.21) holds with any $c \in \mathbb{R}$ such that $c \geq 0$ and $c_{2} \leq c \leq c_{1}$, e.g., with $c=c_{1}$. Lemma 3.5 yields the desired inequality.

Corollary 3.6 shows that the initial value problem for eq. (3.20) with the initial condition $z(-1)=0$ possesses a unique (absolutely continuous) solution $z \equiv z_{c}:[-1,1] \rightarrow \mathbb{R}$, whenever $c \geq 0$ is a fixed number. A standard combination of compactness (ArzelàAscoli's theorem) and uniqueness from PH. HARTMAn [16, Theorem 2.1, p. 94] guarantees that the solution mapping $c \mapsto z_{c}: \mathbb{R}_{+} \rightarrow C([-1,1])$ is continuous.

Note that, for any $c \leq 0$, an arbitrary (possibly nonunique) solution $z:[-1,1] \rightarrow \mathbb{R}$ to eq. (3.20) with $z(-1)=0$ must satisfy

$$
z(r) \geq \int_{-1}^{r} f(-s) \mathrm{d} s=\int_{-r}^{1} f\left(s^{\prime}\right) \mathrm{d} s^{\prime}=F(1)-F(-r)>0 \quad \text { for every } r \in(-1,1),
$$

by a simple integration of this equation over the interval $[-1,1]$ followed by ineq. (1.4). Assuming $F(1)>0$, we get also $z(1) \geq \int_{-1}^{1} f(-s) \mathrm{d} s=F(1)>0$. 
Consequently, assuming ineq. (1.4) and $F(1)>0$, let us define

$$
c^{*} \stackrel{\text { def }}{=} \sup \left\{c \in \mathbb{R}_{+}: z_{c}(1)>0\right\}
$$

Clearly, $c^{*} \geq 0$. As expected, we will show that precisely $c^{*}$ is the desired critical value of the constant $c$, and $c^{*}>0$. From the continuity of the mapping $c \mapsto z_{c}(1): \mathbb{R}_{+} \rightarrow \mathbb{R}$ combined with $z_{0}(1)>0$ (for $c=0$ ), we deduce that either $c^{*}=+\infty$, or else $0<c^{*}<+\infty$ in which case $z_{c^{*}}(1)=0$ and, consequently, $c^{*}$ is the desired critical value. In what follows we exclude the former case, $c^{*}=+\infty$, which would force $z_{c}(1)>0$ for every $c \in \mathbb{R}_{+}$.

Lemma 3.5 has another important corollary which, under stronger hypotheses on $H$, strengthens the conclusion of Corollary 3.6.

Corollary 3.7. Let $c_{1}, c_{2} \in \mathbb{R}$ satisfy $c_{1}>c_{2}$ and $c_{1} \geq 0$. Assume that $z_{1}, z_{2}:[a, b] \rightarrow \mathbb{R}$ are two absolutely continuous functions on some interval $[a, b] \subset \mathbb{R}$, such that $-1 \leq a<$ $b \leq 1$, and the following equations hold for almost every $r \in(a, b)$ :

$$
\begin{aligned}
& \frac{\mathrm{d} z_{1}}{\mathrm{~d} r}+c_{1} H\left(z_{1}^{+}\right)=f(-r), \\
& \frac{\mathrm{d} z_{2}}{\mathrm{~d} r}+c_{2} H\left(z_{2}^{+}\right)=f(-r) .
\end{aligned}
$$

(i) If $z_{1}(a) \leq z_{2}(a)$ then precisely one of the following two alternatives occurs: either $z_{1}(r)<z_{2}(r)$ or else $z_{1}(r)=z_{2}(r) \leq 0$ for every $r \in(a, b)$.

(ii) If $-\mu \leq a<b \leq 1$ and $z_{1}(a) \leq z_{2}(a) \leq 0$, then $z_{2}(r)-z_{1}(r)=z_{2}(a)-z_{1}(a)$ holds for all $r \in[a, b]$. More precisely, we have

$$
\begin{array}{r}
z_{i}(r)=z_{i}(a)+\int_{a}^{r} f(-s) \mathrm{d} s=z_{i}(a)+\int_{-r}^{-a} f(s) \mathrm{d} s<z_{i}(a) \leq 0 \\
\text { for all } r \in(a, b] ; \quad i=1,2 .
\end{array}
$$

(iii) Assume that $H=\psi \circ \Psi_{-1}$ on $\mathbb{R}_{+}$. If $-\mu \leq a<b \leq 1, z_{1}(a) \leq z_{2}(a)$, and $z_{1}(r)>0$ for all $r \in(a, b)$, then $z_{1}(r)<z_{2}(r)$ holds for all $r \in(a, b]$.

Proof. Part (i): It follows from eqs. (3.26) and (3.27) that both functions $z_{1}, z_{2}$ : $[a, b] \rightarrow \mathbb{R}$ are continuously differentiable. Assuming $z_{1}(a) \leq z_{2}(a)$, from Corollary 3.6 we deduce $z_{1}(r) \leq z_{2}(r)$ for all $r \in[a, b]$. Now suppose there is some $r_{0} \in(a, b)$ such that $z_{1}\left(r_{0}\right)=z_{2}\left(r_{0}\right)>0$. Consequently, we have also $\frac{\mathrm{d}}{\mathrm{d} r}\left(z_{2}-z_{1}\right)\left(r_{0}\right)=0$. We insert the equalities $z_{1}\left(r_{0}\right)=z_{2}\left(r_{0}\right)>0$ and $z_{1}^{\prime}\left(r_{0}\right)=z_{2}^{\prime}\left(r_{0}\right)$ into eqs. (3.26) and (3.27), thus arriving at $c_{1} H\left(z_{1}\left(r_{0}\right)\right)=c_{2} H\left(z_{2}\left(r_{0}\right)\right)$. Since $H\left(z_{1}\left(r_{0}\right)\right)=H\left(z_{2}\left(r_{0}\right)\right)>0$, we conclude that $c_{1}=c_{2}$ which contradicts our hypothesis $c_{1}>c_{2}$.

Part (ii): This part is derived directly from eq. (3.20) considered for $r \in(a, b)$ with an unknown function $z:[a, b] \rightarrow \mathbb{R}$ that is assumed to be absolutely continuous. Assuming $-\mu \leq a<b \leq 1$ and $z(a) \leq 0$, for every $r \in(a, b)$ we have $-1 \leq-b<-r<-a \leq \mu$ which yields $f(-r)<0$ and, consequently, by eq. (3.20), $z:[a, b] \rightarrow \mathbb{R}$ is a strictly 
monotone decreasing function satisfying

$$
\begin{array}{r}
z(r)=z(a)+\int_{a}^{r} f(-s) \mathrm{d} s=z(a)+\int_{-r}^{-a} f(s) \mathrm{d} s<z(a) \leq 0 \\
\quad \text { for all } r \in(a, b] .
\end{array}
$$

Notice that, in this part, the value of the constant $c \in \mathbb{R}$ is completely irrelevant.

Part (iii): Next, assume $-\mu \leq a<b \leq 1, z_{1}(a) \leq z_{2}(a)$, and $z_{1}(r)>0$ for all $r \in(a, b)$. Then also $z_{2}(r) \geq z_{1}(r)>0$ holds for all $r \in(a, b)$, by Corollary 3.6. Therefore, we can take advantage of the substitution $y=\Psi(v)$ defined in eq. (3.14), combined with $z(r)=y(-r)$ and $w(r)=v(-r)$ for $r \in[-1,1]$, and use eq. (3.17) for $v(r)$ in place of (3.15) for $y(r)$. For the unknown function $w=w(r)=v(-r)$ in place of $v, w=\Psi_{-1}(z)$, eq. (3.17) becomes

$$
\frac{\mathrm{d} w}{\mathrm{~d} r}=-c+\frac{f(-r)}{\psi(w)}, \quad r \in(-1,1),
$$

with the boundary conditions

$$
w(-1)=w(1)=0 .
$$

Function $w_{i}=\Psi_{-1}\left(z_{i}\right)$ satisfies eq. (3.30) with $c_{i}$ in place of $c$; for $i=1,2$. We subtract (3.30) for $i=1$ from (3.30) for $i=2$, thus arriving at

$$
\begin{aligned}
& \frac{\mathrm{d}}{\mathrm{d} r}\left(w_{2}(r)-w_{1}(r)\right)=-c_{2}+\frac{f(-r)}{\psi\left(w_{2}(r)\right)}+c_{1}-\frac{f(-r)}{\psi\left(w_{1}(r)\right)} \\
& =\left(c_{1}-c_{2}\right)-\frac{f(-r)}{\psi\left(w_{1}(r)\right) \psi\left(w_{2}(r)\right)}\left[\psi\left(w_{2}(r)\right)-\psi\left(w_{1}(r)\right)\right] \geq c_{1}-c_{2},
\end{aligned}
$$

thanks to $\psi\left(w_{2}(r)\right) \geq \psi\left(w_{1}(r)\right)>0$ and $f(-r)<0$ for every $r \in(a, b)$, i.e., $-r \in$ $(-b,-a) \subset(-1, \mu)$. Equivalently,

$$
r \longmapsto\left(w_{2}(r)-w_{1}(r)\right)-\left(c_{1}-c_{2}\right) r:[a, b] \rightarrow \mathbb{R}
$$

is a monotone increasing, continuous function. In particular, we have

$$
\begin{array}{r}
\left(w_{2}(r)-w_{1}(r)\right)-\left(c_{1}-c_{2}\right)(r-a) \geq\left(w_{2}(a)-w_{1}(a)\right) \geq 0 \\
\text { for all } r \in[a, b] .
\end{array}
$$

Since $c_{1}>c_{2}$, this shows that $w_{2}(r)>w_{1}(r)$ holds for all $r \in(a, b]$. Finally, function $\left.\Psi\right|_{\mathbb{R}_{+}}: \mathbb{R}_{+} \rightarrow \mathbb{R}$ being continuous and strictly monotone increasing, we conclude that $z_{2}(r)=\Psi\left(w_{2}(r)\right)>z_{1}(r)=\Psi\left(w_{1}(r)\right)$ holds for all $r \in(a, b]$, as claimed.

Our corollary is proved.

Now let us return to eq. (3.19) with $H=\psi \circ \Psi_{-1}$ on $\mathbb{R}_{+}$, that is, to our original equation, eq. (3.15),

$$
\frac{\mathrm{d} y}{\mathrm{~d} r}-c \psi\left(\Psi_{-1}\left(y^{+}\right)\right)+f(r)=0, \quad r \in(-1,1) .
$$


The uniqueness of the critical speed $c \in \mathbb{R}$, for which eq. (3.15) possesses a positive solution $y:(-1,1) \rightarrow \mathbb{R}$ satisfying the Dirichlet boundary conditions $\lim _{r} \searrow-1 y(r)=0$ and $\lim _{r \nearrow+1} y(r)=0$, follows from the following proposition stated and proved for $z(r)=$ $y(-r), r \in[-1,1]$.

Proposition 3.8. Let $H=\psi \circ \Psi_{-1}$ on $\mathbb{R}_{+}$and let $c_{1}, c_{2} \in \mathbb{R}$ satisfy $c_{1} \geq c_{2}$ and $c_{1} \geq 0$. Assume that $z_{1}, z_{2}:[-1,1] \rightarrow \mathbb{R}$ are two absolutely continuous functions satisfying the differential equations (3.26) and (3.27), respectively, such that $z_{1}(r)>0$ for all $r \in$ $(-1,1)$, together with $z_{i}(-1)=z_{i}(1)=0$ for $i=1,2$. Then we must have $c_{1}=c_{2}$ and $z_{1} \equiv z_{2}$ in $[-1,1]$.

Proof. On the contrary, suppose that $c_{1}>c_{2}$ is possible. Corollary 3.7, Part (i), with $a=-1$ and $b=1$, implies that $z_{1}(r)<z_{2}(r)$ for every $r \in(-1,1)$. Part (iii) forces also $z_{1}(1)<z_{2}(1)$, a contradiction with our boundary conditions $z_{1}(1)=z_{2}(1)=0$.

We have proved $c_{1}=c_{2}$. The equality $z_{1} \equiv z_{2}$ in $[-1,1]$ follows from Corollary 3.6 with $c_{1}=c_{2}$.

Finally, the following lemma excludes the case $c^{*}=+\infty$ in eq. (3.25). Consequently, the existence of the critical speed $c \in \mathbb{R}$ follows from eq. (3.25) and remarks thereafter, $c=c^{*} \in(0, \infty)$.

Lemma 3.9. We have $\lim _{c \rightarrow+\infty} z_{c}(1)<0$.

Proof. By Corollary 3.7, Part (i), with $a=-1$ and $b=1$, the monotone decreasing $\operatorname{limit} L=\lim _{c \rightarrow+\infty} z_{c}(1)$ exists, i.e., $z_{c}(1) \searrow L$ as $c \nearrow+\infty$, and satisfies $-\infty \leq L \leq$ $z_{0}(1)<\infty$. On the contrary, suppose that $L \geq 0$. This forces $z_{c}(1) \geq L \geq 0$ for every $c \geq 0$.

Given any $c>0$, we must have $z_{c}(r)>0$ for every $r \in(-1,-\mu)$. To verify this claim, we first show that, for every $\delta \in(0,1-\mu)$ there is some $r_{\delta} \in(-1,-1+\delta)$, such that $z_{c}\left(r_{\delta}\right)>0$. Namely, otherwise we would have $z_{c}(r) \leq 0$ for every $r \in(-1,-1+\delta)$ and, consequently, $z_{c}^{\prime}(r) \equiv \frac{\mathrm{d}}{\mathrm{d} r} z_{c}(r)=f(-r)>0$, by eq. (3.20), which in turn yields $z_{c}(r)>0$ for every $r \in(-1,-1+\delta)$, a contradiction. Again, notice that $r \in(-1,-1+\delta)$ means $-r \in(1-\delta, 1) \subset(\mu, 1)$ whence $f(-r)>0$. Next, we show that $z_{c}(r)>0$ for every $r \in\left[r_{\delta},-\mu\right)$. Indeed, if $z_{c}\left(r^{\prime}\right)=0$ for some $r^{\prime} \in\left(r_{\delta},-\mu\right)$, then there is another number $r^{\prime \prime} \in\left(r_{\delta}, r^{\prime}\right]$, such that $z_{c}\left(r^{\prime \prime}\right)=0$ and $z_{c}(r)>0$ for every $r \in\left[r_{\delta}, r^{\prime \prime}\right)$. But this forces $z_{c}^{\prime}\left(r^{\prime \prime}\right) \leq 0$ which contradicts $z_{c}^{\prime}\left(r^{\prime \prime}\right)=f\left(-r^{\prime \prime}\right)>0$, by eq. (3.20). Finally, letting $\delta \searrow 0$ we arrive at $z_{c}(r)>0$ for every $r \in(-1,-\mu)$.

Since $c>0$ and $f(r)<0$ whenever $r \in(-1, \mu)$, it follows from eq. (3.20) that $z_{c}^{\prime}(r) \leq f(-r)<0$ for all $r \in(-\mu, 1)$. Consequently, $z_{c}:[-1,1] \rightarrow \mathbb{R}$ is strictly monotone decreasing on the interval $[-\mu, 1]$. Recalling $z_{c}(1) \geq L \geq 0$ and $z_{c}(r)>0$ for every $r \in(-1,-\mu)$, we conclude that $z_{c}(r)>0$ for every $r \in(-1,1)$. Now the positivity of $z_{c}$ on the interval $(-1,1)$ enables us to invoke eq. (3.30) for the unknown function 
$w \equiv w_{c}=\Psi_{-1}\left(z_{c}\right)$ on $(-1,1)$ with the initial condition $w(-1)=0$,

$$
\frac{\mathrm{d} w}{\mathrm{~d} r}=-c+\frac{f(-r)}{\psi(w)}, \quad r \in(-1,1) .
$$

Here, $w_{c}(r)=\Psi_{-1}\left(z_{c}(r)\right)>0$ for all $r \in(-1,1)$. In particular, we have $w_{c}^{\prime}(r)<-c$ for every $r \in(-\mu, 1)$. Taking $c>0$ sufficiently large, say, $c \geq c_{0}>0$, we conclude that

$$
\begin{array}{r}
w_{c}(-\mu+s)=w_{c}(-\mu)+\int_{-\mu}^{-\mu+s} w_{c}^{\prime}(r) \mathrm{d} r<w_{c}(-\mu)-c s \leq w_{c}(-\mu)-c_{0} s \leq 0 \\
\text { whenever } \quad \frac{w_{c}(-\mu)}{c_{0}} \leq s<1+\mu .
\end{array}
$$

But this contradicts the fact that $w_{c}(r)>0$ for all $r \in(-1,1)$.

We have proved $L<0$ as desired.

Proposition 3.1 follows directly from a combination of Lemma 3.9 (existence) and Proposition 3.8 (uniqueness). In addition, Lemma 3.2 is a consequence of the continuous dependence of the travelling wave $q$ and the speed $c$ upon the given data, combined with a standard compactness argument (Arzelà-Ascoli's theorem), by a continuity (convergence) result from PH. HARTMAN [16, Theorem 2.1, p. 94] for problem (1.14), (1.15).

\section{Convergence to the limit problem}

In analogy with the approach in E. FEIREISL [12], our proof of Theorem 2.6 is based on a comparison principle. To begin with, we introduce an approximation family $\left\{\Phi_{\alpha}\right\}_{\alpha>0}$ satisfying $(2.9)-(2.13)$. We start with a simple result for $N=1$.

Lemma 4.1. Suppose that $v_{\alpha}$ is a weak solution of the Cauchy problem (1.1), (1.2) with $N=1$ and $\Phi=\Phi_{\alpha}$ starting from (smooth) initial data $v_{0}$,

$$
\begin{gathered}
\partial_{x} v_{0} \leq 0, v_{0}(x)=\lambda_{1} \text { for all } x<a, v_{0}(x)=\lambda_{2} \\
\text { for all } x>b, a<b, \lambda_{1} \in(\mu, 1], \lambda_{2} \in[-1, \mu) .
\end{gathered}
$$

Then

$$
\lim _{t \rightarrow \infty} v_{\alpha}(t, \underline{c} t)=1 \text { for any } \underline{c}<c, \quad \lim _{t \rightarrow \infty} v_{\alpha}(t, \bar{c} t)=-1 \quad \text { for any } \bar{c}>c,
$$

uniformly for $\alpha \searrow 0$.

Remark 4.2. Uniformly in (4.1) means that there exists $\alpha_{0}=\alpha_{0}(\underline{c}, \bar{c})>0$ such that for any $\varepsilon>0$ there exist $T(\varepsilon)>0$ such that

$$
v_{\alpha}(t, \underline{c} t)>1-\varepsilon, v_{\alpha}(t, \bar{c} t)<-1+\varepsilon \text { for all } \alpha<\alpha_{0}(\underline{c}, \bar{c}), t>T(\varepsilon) .
$$

Proof: In view of the symmetry of the problem with respect to the change $v \approx-v$, it is enough to show

$$
\lim _{t \rightarrow \infty} v_{\alpha}(t, \underline{c} t)=1 \text { for any } \underline{c}<c, \text { uniformly for } \alpha \searrow 0,
$$


for

$\partial_{x} v_{0} \leq 0, v_{0}=v_{0}(x)=\lambda_{1}$ for all $x<a, v_{0}(x)=-1$ for all $x>b, a<b, \lambda_{1} \in(\mu, 1)$.

Step 1. We show that for any $\varepsilon>0$ there exist a time $T(\varepsilon), a(\varepsilon)$, and $\alpha_{0}>0$ such that

$$
v_{\alpha}(T(\varepsilon), x)>1-\varepsilon \text { for all } x<a(\varepsilon), \alpha<\alpha_{0} .
$$

By the comparison principle, the spatially homogeneous solution $\bar{v}=\bar{v}(t)$,

$$
\partial_{t} \bar{v}(t)=f(\bar{v}(t)), \bar{v}(0)=\lambda_{1}
$$

dominates $v_{\alpha}$,

$$
\bar{v}(t) \geq v_{\alpha}(x, t) \text { for all } t \geq 0, x \in R, \alpha>0 .
$$

Since $f$ satisfies (1.3), we have

$$
\bar{v}(t) \rightarrow 1 \quad \text { as } t \rightarrow \infty
$$

Given parameters $\delta \in(0, \infty)$ and $Y \in \mathbb{R}$, let us consider an auxiliary function $\omega_{\delta, Y}$ : $\mathbb{R} \rightarrow[0,1]$ defined for every $x \in \mathbb{R}$ by $\omega_{\delta, Y}(x) \stackrel{\text { def }}{=} \omega_{\delta, 0}(x-Y)$ where

$$
\omega_{\delta, 0}(x) \stackrel{\text { def }}{=}\left\{\begin{array}{cl}
1 & \text { if }|x| \leq \frac{1}{2} \\
\delta\left(\frac{1}{2}+\frac{1}{\delta}-|x|\right) & \text { if } \frac{1}{2}<|x| \leq \frac{1}{2}+\frac{1}{\delta} \\
0 & \text { if } \frac{1}{2}+\frac{1}{\delta}<|x|<\infty .
\end{array}\right.
$$

Clearly, $\omega_{\delta, 0}$ is an even function (about 0 ), i.e., $\omega_{\delta, 0}(x)=\omega_{\delta, 0}(|x|)$ for all $x \in \mathbb{R}$.

Using equation (1.1) with $\Phi$ replaced by $\Phi_{\alpha}$, we get

$$
\int_{R} \omega_{\delta, Y}\left(\bar{v}-v_{\alpha}\right)(\tau) \mathrm{d} x
$$

$\leq \int_{R} \omega_{\delta, Y}\left(\bar{v}(0)-v_{0}\right) \mathrm{d} x+\int_{0}^{\tau} \int_{R} \omega_{\delta, Y}\left|f(\bar{v})-f\left(v_{\alpha}\right)\right| \mathrm{d} x \mathrm{~d} t+\int_{0}^{\tau} \int_{R} \partial_{z} \varphi_{\alpha}\left(\left|\partial_{x} v_{\alpha}\right|\right)\left|\partial_{x} \omega_{\delta, Y}\right| \mathrm{d} x \mathrm{~d} t$ for any $\tau \geq 0$.

In accordance with Corollary 2.3, there is $M, \alpha_{0}>0$ such that

$$
\left|\partial_{x} v_{\alpha}(t, x)\right| \leq M \quad \text { for all } t \geq 0, x \in \mathbb{R}^{N}, \alpha<\alpha_{0} .
$$

Moreover, by virtue of the hypothesis (2.3), we get

$$
\begin{array}{r}
\mathrm{d}_{z} \log \left(z^{\Lambda_{1}}\right)=\frac{\Lambda_{1}}{z} \leq \mathrm{d}_{z}\left(\log \mathrm{d}_{z} \varphi(z)\right) \leq \frac{\Lambda_{2}}{z}=\mathrm{d}_{z} \log \left(z^{\Lambda_{2}}\right) \\
\text { for all } z \in(0, \infty),
\end{array}
$$

which upon integration shows that, with some constant $m \in(0,1)$, on every compact interval $[0, M] \subset \mathbb{R}_{+}$we have

$$
0 \leq \partial_{z} \varphi_{\alpha}(z) \leq \operatorname{const}(M)\left(z+z^{m}\right) \quad \text { for all } 0 \leq z \leq M, \alpha<\alpha_{0} ;
$$


hence

$$
\left\|\partial_{z} \varphi_{\alpha}\left(\left|\partial_{x} v_{\alpha}\right|\right)\right\|_{L^{1}+L^{q}(\mathbb{R})} \leq \operatorname{const}(M)\left(\left\|\partial_{x} v_{\alpha}\right\|_{L^{1}(\mathbb{R})}+\left\|\partial_{x} v_{\alpha}\right\|_{L^{1}(\mathbb{R})}^{m}\right), \quad q=\frac{1}{m} .
$$

Since $\partial_{x} v_{\alpha} \leq 0$, we get

$$
\left\|\partial_{x} v_{\alpha}(t, \cdot)\right\|_{L^{1}(\mathbb{R})} \leq 2 \text { for all } t \geq 0, \alpha<\alpha_{0},
$$

thus yielding

$$
\left\|\partial_{z} \varphi_{\alpha}\left(\left|\partial_{x} v_{\alpha}\right|\right)\right\|_{L^{1}+L^{q}(\mathbb{R})} \leq \operatorname{const}(M), q=\frac{1}{m} .
$$

We remark that the sum $L^{1}+L^{q}(\mathbb{R}) \equiv L^{1}(\mathbb{R})+L^{q}(\mathbb{R})$ and its norm are defined in a standard way used in interpolation theory, cf. H. TRIEBEL [23, §1.2.1].

On the other hand,

$$
\left\|\partial_{x} \omega_{\delta, Y}\right\|_{L^{\infty}(R)}=\delta, \quad\left\|\partial_{x} \omega_{\delta, Y}\right\|_{L^{1}(R)}=2 ;
$$

therefore, going back to (4.4), we may infer that

$$
\int_{R} \omega_{\delta, Y}\left(\bar{v}-v_{\alpha}\right)(\tau) \mathrm{d} x \leq \exp \left(L_{f} \tau\right)\left(\int_{R} \omega_{\delta, Y}\left(\bar{v}(0)-v_{0}\right) \mathrm{d} x+\tau \chi(\delta)\right),
$$

where $L_{f}$ is the Lipschitz constant of $f$ and $\chi(\delta) \rightarrow 0$ for $\delta \searrow 0$.

In accordance with (4), we may fix $\tau=T(\varepsilon)$ in (4.5) so that

$$
\bar{v}(t)>\left(1-\frac{\varepsilon}{2}\right) \text { for all } t>\tau,
$$

and take $\delta>0$ so small and $Y<0$ so that (4.5) yields the existence of a point $a(\varepsilon)$ such that

$$
v_{\alpha}(T(\varepsilon), a(\varepsilon))>1-\varepsilon, \alpha<\alpha_{0} .
$$

As $\partial_{x} v_{\alpha} \leq 0$, the desired conclusion (4.3) follows.

Step 2. In agreement with the previous discussion, it is enough to examine the initial datum

$$
\partial_{x} v_{0} \leq 0, v_{0}(x)=1-\varepsilon \text { for all } x<a, v_{0}(x)=-1 \text { for all } x>b, a<b,
$$

where $\varepsilon \searrow 0$.

Keeping the conclusion of Lemma 3.2 in mind, we take a family $f_{\varepsilon} \approx f$ satisfying the hypotheses of Lemma 3.2 with

$$
b_{\varepsilon}=1-\varepsilon, a_{\varepsilon}=-1-\varepsilon, f_{\varepsilon} \leq f .
$$

Consequently, taking $\alpha_{0}(\underline{c})>0$ small enough, we can find a traveling wave $q_{\alpha}$ with the propagation speed $c_{\alpha}$,

$$
c>c_{\alpha}>\underline{c} \text { for all } \alpha<\alpha_{0}(\underline{c})
$$

such that

$$
q_{\alpha}(x+D) \leq v_{0}(x) \text { for all } x \in R
$$

for a suitable constant $D \in R$, where, by comparison, $v_{\alpha}(t, x) \geq q_{\alpha}\left(x+D-c_{\alpha} t\right)$, in particular, $v_{\alpha}(t, \underline{c} t) \geq q_{\alpha}\left(D+\left(\underline{c}-c_{\alpha}\right) t\right) \rightarrow 1-\varepsilon$ as $t \rightarrow \infty$ 
uniformly for $\alpha<\alpha_{0}$.

Thus we have shown

$$
\liminf _{t \rightarrow \infty} v_{\alpha}(t, \underline{c} t) \geq 1-\varepsilon \text { uniformly for } \alpha<\alpha_{0} .
$$

Since $\varepsilon>0$ can be taken arbitrarily small, the desired conclusion follows.

The next step is to extend the previous result to the case of radially symmetric data in $\mathbb{R}^{N}$. To this end, we introduce a new variable $r=|x|$ and rewrite (formally) equation (1.1) for the radially symmetric solutions:

$$
\begin{array}{r}
\partial_{t} u=\partial_{r}\left(\partial \varphi\left(\left|\partial_{r} u\right|\right) \frac{\partial_{r} u}{\left|\partial_{r} u\right|}\right)+\frac{N-1}{r} \partial \varphi\left(\left|\partial_{r} u\right|\right) \frac{\partial_{r} u}{\left|\partial_{r} u\right|}+f(u), \\
u=u(t, r), r>0, \partial_{r} u(t, 0)=0 .
\end{array}
$$

Here, we have used the following simple relations for the radially symmetric functions $\Phi(Z) \equiv \varphi(|Z|), Z \in \mathbb{R}^{N}$, and $u(x, t) \equiv u(r, t), x \in \mathbb{R}^{N}, r=|x|$ :

$$
\begin{aligned}
& \partial \Phi(Z)=\partial \varphi(|Z|) \frac{Z}{|Z|} \quad \text { and } \quad \nabla_{x} u(x, t)=\partial_{r} u(r, t) \frac{x}{r} \quad \text { for } Z, x \in \mathbb{R}^{N} \backslash\{\mathbf{0}\}, \\
& \nabla_{x}(|x|)=\frac{x}{|x|} \quad \text { and } \quad \operatorname{div}\left(\frac{x}{|x|}\right)=\frac{N-1}{|x|} \quad \text { for } \quad x \in \mathbb{R}^{N} \backslash\{\mathbf{0}\} .
\end{aligned}
$$

Lemma 4.3. Suppose that $v_{\alpha}=v_{\alpha}(t, r)$ is the radially symmetric weak solution of the Cauchy problem (1.1), (1.2), with $\Phi_{\alpha}$ emanating from (smooth) initial datum $v_{0}=v_{0}(r)$,

$$
\begin{aligned}
& \partial_{r} v_{0} \leq 0, v_{0}(r)=\lambda \quad \text { for all } r \in(0, R), \\
& v_{0}(r) \geq-1 \quad \text { for all } r \in(\tilde{R}, \infty), 0<R<\tilde{R}, \quad \text { with } \lambda \in\left(\lambda_{0}, 1\right), \lambda_{0} \in(\mu, 1) .
\end{aligned}
$$

Then for any $\varepsilon>0$ and $\underline{c}<c$, there exists $\alpha=\alpha_{0}(\underline{c})$, a time $T=T\left(\lambda_{0}, \varepsilon, \underline{c}\right)$ and $R_{0}=R_{0}\left(\lambda_{0}, \varepsilon, \underline{c}\right)$ such that

$$
v_{\alpha}(t, r) \geq 1-\varepsilon \text { for all } t \in[T, 2 T],|x|<R+\underline{c} t, \alpha<\alpha_{0},
$$

whenever $R>R_{0}$.

\section{Proof:}

The proof is along the same lines as that of Lemma 4.1. Assuming $\delta>0$ is chosen small enough, we fix a (smooth) profile

$$
w_{0}=w_{0}(x), \partial_{x} w_{0} \leq 0, w_{0}(x)=\lambda \text { for } x<0, w_{0}(x)=-1 \text { for } x>1,
$$

and such that

$$
w_{0}(r-R) \leq v_{0}(r) \text { for all } r>0 .
$$

By virtue of the comparison principle, it is enough to show the conclusion of the lemma for $v_{0}(r)=w_{0}(r-R)$. 
Step 1. Consider the unique solution $w_{\alpha}$ of the Cauchy problem

$$
\partial_{t} w_{\alpha}=\partial_{x}\left(\partial_{z} \varphi_{\alpha}\left(\left|\partial_{x} w_{\alpha}\right|\right) \frac{\partial_{x} w_{\alpha}}{\left|\partial_{x} w_{\alpha}\right|}\right)+f\left(w_{\alpha}\right), t>0, x \in R^{1}, w_{\alpha}(0, x)=w_{0}(x) .
$$

Making use once more of the comparison principle we deduce that

$$
w_{\alpha}(t, r-R) \geq v_{\alpha}(t, r) \text { for any } r>0 \text { and } t \geq 0,
$$

where, by virtue of Lemma 4.1,

$$
\lim _{t \rightarrow \infty} \inf _{r<\underline{c} t} w_{\alpha}(t, r)=1 \text { for any } \underline{c}<c \text { uniformly in } \alpha<\alpha_{0}(\underline{c}) .
$$

Step 2. Similarly to the proof of Lemma 4.1, we take the function $\omega_{\delta, Y}$ and compute the "distance"

$$
\begin{gathered}
\int_{0}^{\infty} \omega_{\delta, Y}\left[w_{\alpha}(\tau, r-R)-v_{\alpha}(\tau, r)\right] \mathrm{d} r \leq \int_{0}^{\tau} \int_{0}^{\infty} \omega_{\delta, Y}\left|f\left(w_{\alpha}(t, r-R)\right)-f\left(v_{\alpha}(t, r)\right)\right| \mathrm{d} r \mathrm{~d} t \\
+\int_{0}^{\tau} \int_{0}^{\infty}\left(\partial_{z} \varphi_{\alpha}\left(\left|\partial_{r} v_{\alpha}(t, r)\right|\right)+\partial_{z} \varphi_{\alpha}\left(\left|\partial_{r} w_{\alpha}(t, r-R)\right|\right)\right)\left|\partial_{r} \omega_{\delta, Y}\right| \mathrm{d} r \mathrm{~d} t \\
+\int_{0}^{\tau} \int_{0}^{\infty} \frac{N-1}{r} \omega_{\delta, Y} \partial_{z} \varphi_{\alpha}\left(\left|\partial_{r} v_{\alpha}(t, r)\right|\right) \mathrm{d} r \mathrm{~d} t
\end{gathered}
$$

for $Y>1+\frac{1}{\delta}$.

Now, exactly as in the proof of Lemma 4.1, we may show that

$\left|\int_{0}^{\tau} \int_{0}^{\infty}\left(\partial_{z} \varphi_{\alpha}\left(\left|\partial_{r} v_{\varepsilon}(t, r)\right|\right)+\partial_{z} \varphi_{\alpha}\left(\left|\partial_{r} w_{\varepsilon}(t, r-R)\right|\right)\right)\right| \partial_{r} \omega_{\delta, Y}|\mathrm{~d} r \mathrm{~d} t| \leq \tau \chi(\delta)$, with $\chi(\delta) \rightarrow 0$ as $\delta \searrow 0$ uniformly for $\alpha<\alpha_{0}$.

$\int_{0}^{\infty} \omega_{\delta, Y}\left[w_{\alpha}(\tau, r-R)-v_{\alpha}(\tau, r)\right] \mathrm{d} r \leq \exp \left(L_{f} \tau\right)\left(\tau \chi(\delta)+\int_{0}^{\tau} \int_{0}^{\infty} \frac{N-1}{r} \omega_{\delta, Y} \partial_{z} \varphi_{\alpha}\left(\left|\partial_{r} v_{\alpha}(t, r)\right|\right) \mathrm{d} r \mathrm{~d} t\right)$ for $\tau>0, Y>1+\frac{1}{\delta}$.

Step 3. In accordance with (4.7), there exists $T=T\left(\lambda_{0}, \varepsilon, \underline{c}\right)$ such that

$$
w_{\alpha}(t, r-R) \geq 1-\frac{\varepsilon}{4} \text { for all } t>T \text { and } r<R+\underline{c} t+2, \alpha<\alpha(\underline{c}) \text {. }
$$

Next, fix $\delta>0$ so that

$$
2 \chi(\delta) T \exp \left(2 L_{f} T\right) \leq \frac{\varepsilon}{4}
$$

Finally, take $Y \geq Y_{0}(\delta)$ so large that

$$
\int_{0}^{2 T} \int_{0}^{\infty} \frac{N-1}{r} \omega_{\delta, Y} \partial_{z} \varphi_{\alpha}\left(\left|\partial_{r} v_{\alpha}(t, r)\right|\right) \mathrm{d} r \mathrm{~d} t \leq \frac{\varepsilon}{4}, \quad Y \geq Y_{0} .
$$


Thus, for $R \geq R_{0}$ large enough so that

$$
Y=R+\underline{c} \tau+1>Y_{0} \text { for all } \tau \in[T, 2 T]
$$

in the inequality $(4.8)$, we may use $(4.9-4.11)$, together with the monotonicity of $v_{\alpha}$ in $r$, to obtain the desired conclusion.

Applying Lemma 4.3 recursively on the sequence of time intervals $[n T,(n+2) T]$ we obtain:

Corollary 4.1. Suppose that $v_{\alpha}=v_{\alpha}(t, r)$ is the radially symmetric weak solution of the Cauchy problem (1.1), (1.2), with $\Phi_{\alpha}$, emanating from (smooth) initial datum $v_{0}=v_{0}(r)$,

$\partial_{r} v_{0} \leq 0, v_{0}(r)=\lambda \quad$ for all $r \in(0, R)$,

$v_{0}(r) \geq-1 \quad$ for all $r \in(\tilde{R}, \infty), 0<R<\tilde{R}, \quad$ with $\lambda \in\left(\lambda_{0}, 1\right), \lambda_{0} \in(\mu, 1)$.

Then for any $\varepsilon>0$ and $\underline{c}<c$, there exist $\alpha_{0}=\alpha_{0}(\underline{c})$, a time $T=T\left(\lambda_{0}, \varepsilon, \underline{c}\right)$ and $R_{0}=R_{0}\left(\lambda_{0}, \varepsilon, \underline{c}\right)$ such that

$$
v_{\alpha}(\tau, r) \geq 1-\varepsilon \text { for all } \tau>T, 0<r<R+\underline{c} \tau, \alpha<\alpha_{0},
$$

as long as

$$
R+\underline{c} t>R_{0} \text { for all } t \in[0, \tau]
$$

Introducing $u_{\varepsilon}=u\left(\frac{t}{\varepsilon}, \frac{x}{\varepsilon}\right)$, the function $u_{\varepsilon}$ solves the scaled equation (1.5). Adapting Corollary 4.1 we get:

Corollary 4.2. Suppose that $\left\{u_{\alpha, \varepsilon}\right\}_{\varepsilon>0}$ is a family of solution of the scaled equation (1.5), with $\Phi=\Phi_{\varepsilon}$, emanating from the initial data

$$
-1 \leq u_{\varepsilon, \alpha}(0, \cdot) \leq 1, u_{\varepsilon, \alpha}(0, x) \geq \lambda \in(\mu, 1] \text { for all }|x|<R .
$$

Then, given a compact set

$$
\mathcal{K} \subset\{t>0,|x|<R+c t\}
$$

there is $\alpha_{0}(\mathcal{K})>0$ such that

$$
\lim _{\varepsilon \rightarrow 0} u_{\varepsilon, \alpha}(t, x)=1 \text { uniformly in } \mathcal{K} \text { and uniformly for } \alpha<\alpha_{0}(\mathcal{K}) .
$$

Finally, using "symmetric" arguments we get

Corollary 4.3. Suppose that $\left\{u_{\alpha, \varepsilon}\right\}_{\varepsilon>0}$ is a family of solution of the scaled equation (1.5), with $\Phi=\Phi_{\varepsilon}$, emanating from the initial data

$$
-1 \leq u_{\varepsilon, \alpha}(0, \cdot) \leq 1, u_{\varepsilon, \alpha}(0, x) \leq \lambda \in[-1, \mu) \text { for all }|x|<R .
$$

Then, given a compact set

$$
\mathcal{K} \subset\{t>0,|x|<R-c t\}
$$


there is $\alpha_{0}(\mathcal{K})>0$ such that

$$
\lim _{\varepsilon \rightarrow 0} u_{\varepsilon, \alpha}(t, x)=-1 \text { uniformly in } \mathcal{K} \text { and uniformly for } \alpha<\alpha_{0}(\mathcal{K}) .
$$

The final observation is that Corollaries 4.2, 4.3 imply the conclusion of Theorem 2.6. The proof is exactly the same as in [12, Section 5] provided we consider only those weak solutions that have been suitably introduced in Definition 2.5.

\section{REFERENCES}

1. D. G. Aronson and H. F. Weinberger, Multidimensional nonlinear diffusion arising in population genetics, Advances in Math., 30 (1978), 33-76.

2. V. Barbu, "Nonlinear Semigroups and differential equations in Banach spaces". Noordhoff International Publising, Leyden, The Netherlands, 1976.

3. G. Barles, L. Bronsard, and P. E. Souganidis, Front propagation for reaction-diffusion equations of bistable type, Ann. Inst. Henri Poincaré (Analyse non Linéaire), 9(5) (1992), 479-496.

4. D. Breit, B. Stroffolini, and A. Verde, A general regularity theorem for functionals with $\phi$-growth, J. Math. Anal. Appl., 383(1) (2011), 226-233.

5. H. Brézis, "Opérateurs maximaux monotones et semi-groupes de contractions dans les espaces de Hilbert". North-Holland Publ. Co., Amsterdam, The Netherlands, 1973.

6. X.-F. Chen, Generation and propagation of interfaces for reaction-diffusion equations, J. Differential Equations, 96 (1992), 116-141.

7. M. G. Crandall, H. Ishii, and P.-L. Lions, User's guide to viscosity solutions of second order partial differential equations, Bulletin (N.S.) Amer. Math. Soc, 27(1) (1992), 1-67.

8. E. DiBenedetto and M. A. Herrero, Nonnegative solutions of the evolution p-Laplacian equation: Initial traces and Cauchy problem when $1<p<2$, Arch. Rational Mech. Anal., 111(3) (1990), $225-290$.

9. E. DiBenedetto and M. A. Herrero, On the Cauchy problem and initial traces for a degenerate parabolic equation, Trans. Amer. Math. Soc., 314(1) (1989), 187-224.

10. I. Ekeland and R. Temam, "Convex Analysis and Variational Problems", North Holland-American Elsevier, Amsterdam-New York, 1976.

11. R. Enguiça, A. Gavioli, and L. Sanchez, A class of singular first order differential equations with applications in reaction-diffusion, Discr. Cont. Dynam. Systems, 33(1) (2013), 173-191.

12. E. Feireisl, Front propagation for degenerate parabolic equations, Nonlinear Analysis, T.M.A., 35 (1999), 735-746.

13. P. C. Fife and J. B. McLeod, The approach of solutions of nonlinear diffusion equations to travelling front solutions, Arch. Rational Mech. Anal., 65(4) (1977), 335-361.

14. R. A. Fisher, The advance of advantageous genes, Ann. of Eugenics, 7 (1937), 355-369.

15. Y. Giga, S. Goto, H. Ishii, and M.-H. Sato, Comparison principle and convexity preserving properties for singular degenerate parabolic equations on unbounded domains, Indiana Univ. Math. J., 40 (1991), 443-470.

16. Ph. Hartman, "Ordinary Differential Equations", 2nd Ed., Birkhäuser, Boston-Basel-Stuttgart, 1982. 
17. P. Juutinen, P. Linqvist, and J. J. Manferdi, On the equivalence of viscosity solutions and weak solutions for a quasilinear equation, SIAM J. Math. Anal., 33(3) (2001), 699-717.

18. A. Kolmogorov, I. Petrovski, and N. Piscounov, Ètude de l'équation de la diffusion avec croissance de la quantité de la matière et son application à un problème biologique, Bull. Univ. Moskou, Ser. Internat., Sec. A, 1 (1937), 1-25.

19. O. A. Ladyzhenskaya, V. A. Solonnikov, and N. N. Ural'tseva, "Linear and Quasi-linear Equations of Parabolic Type", in Transl. Math. Monogr., Vol. 23, American Mathematical Society, Providence, R.I., 1968.

20. G. M. Lieberman, Boundary and initial regularity for solutions of degenerate parabolic equations, Nonlinear Anal., 20(5) (1993), 551-569.

21. G. M. Lieberman, Hölder regularity for the gradients of solutions of degenerate parabolic systems, Ukr. Mat. Visn., 3(3) (2006), 352-373.

22. P. E. Souganidis, Front propagation: theory and applications, in "Viscosity Solutions and Applications", Montecatini Terme, 1995. Lecture Notes in Mathematics, Vol. 1660, pp. 186-242. SpringerVerlag, Berlin-Heidelberg-New York, 1997.

23. H. Triebel, "Interpolation Theory, Function Spaces, Differential Operators". North-Holland Publ. Co., New York-Amsterdam-Oxford, 1978.

24. J. N. Zhao and Q. Yi, Generation and propagation of interfaces for p-Laplacian equations, Acta Math. Sinica (English Ser.), 20 (2004), 319-332.

Eduard Feireisl, Institute of Mathematics, Academy of Sciences of the Czech Republic, Žitná 25, CZ - 11567 Praha 1, Czech Republic

E-mail address: feireisl@math.cas.cz

Danielle Hilhorst, CNRS et Laboratoire de Mathématiques, Université de Paris-Sud, BÂtiment 425, FR - 91405 Orsay Cedex, France

E-mail address: Danielle.Hilhorst@math.u-psud.fr

Hana Petzeltová, Institute of Mathematics, Academy of Sciences of the Czech Republic, Žitná 25, CZ - 11567 Praha 1, Czech Republic

E-mail address: petzelt@math.cas.cz

Peter Takáč, Institut für Mathematik, Universität Rostock, Ulmenstrasse 69, Haus 3, D-18055 Rostock, Germany

E-mail address: peter.takac@uni-rostock.de 